\title{
Mathematical models of morphogen gradients and their effects on gene expression
}

\author{
Stanislav Y. Shvartsman ${ }^{1 *}$ and Ruth E. Baker ${ }^{2}$
}

\begin{abstract}
An introduction to mathematical models of pattern formation by morphogen gradients is presented, using the early embryo of the fruit fly Drosophila as the main experimental example. Analysis of morphogen gradient formation is based on the source-diffusion-degradation models and a formalism of local accumulation times. Transcriptional control by morphogens is discussed within the framework of thermodynamic site occupancy models of gene regulatory regions. ๑ 2012 Wiley Periodicals, Inc.
\end{abstract}

How to cite this article:

WIREs Dev Biol 2012. doi: 10.1002/wdev.55

\section{INTRODUCTION}

$\mathrm{M}$ echanisms of developmental pattern formation can be assigned to two broad classes, inductive and self-organized. In inductive mechanisms, the emergence of pattern formation depends on nonuniformities established during previous stages of development. In self-organized mechanisms, patterns emerge through spontaneous symmetry breaking, in a way that is largely independent of preexisting asymmetries. Molecular studies of development have provided examples of both inductive and selforganized mechanisms. For instance, the overall plan of our hand depends on an intricate cascade of inductive events. At the same time, the quasiperiodic array of the hair follicles on the dorsal side of the hand is likely to be generated by a mechanism that is self-organized.

In this review, we focus on a specific class of inductive pattern formation mechanisms, which depend on morphogen gradients, defined as the concentration fields of chemicals that act as dosedependent regulators of cell signaling and gene expression. ${ }^{1-4}$ The term morphogen was introduced in a 1952 paper by Alan Turing to describe chemical

\footnotetext{
*Correspondence to: stas@princeton.edu

${ }^{1}$ Department of Chemical and Biological Engineering and LewisSigler Institute for Integrative Genomics, Princeton University, NJ, USA

${ }^{2}$ Centre for Mathematical Biology, Mathematical Institute, University of Oxford, Oxford, USA
}

substances that 'persuade' cells to acquire a specific identity. ${ }^{5}$ The molecular identities of morphogens remained a mystery until the late 1980s, when the first morphogens were identified by in the early Drosophila embryo, ${ }^{6-11}$ an experimental system that has a simple anatomy and can be studied by powerful genetic techniques.

During the first two hours of Drosophila development, a spatially uniform arrangement of identical cells is patterned by graded distributions of transcription factors, which play a key role in establishing the body plan of the adult fly (Figure 1). The anterior-posterior concentration gradient of Bicoid (Bcd), a transcriptional activator, is essential for specifying the anterior segments of the body. ${ }^{12}$ The ventral-to-dorsal nuclear localization gradient of Dorsal (Dl), a transcription factor that can act both as an activator and as a repressor, specifies the spatial arrangement of the muscle, nerve, and skin tissues. ${ }^{13}$ Graded distribution of a transcriptional repressor Capicua (Cic), with minima at both the anterior and posterior poles, is important for specifying the terminal structures of the embryo. ${ }^{14}$ Graded distributions of $\mathrm{Bcd}$, Cic, and nuclear $\mathrm{Dl}$ provide inputs to the regulatory regions of genes involved in multiple aspects of embryogenesis (Figures 2 and 3).

Each of these gradients arises from asymmetries in the unfertilized egg. The origin of the $\mathrm{Bcd}$ gradient can be traced to the localized spatial pattern of $b c d$ mRNA, which is established during oogenesis. Following egg fertilization, the $b c d$ mRNA 


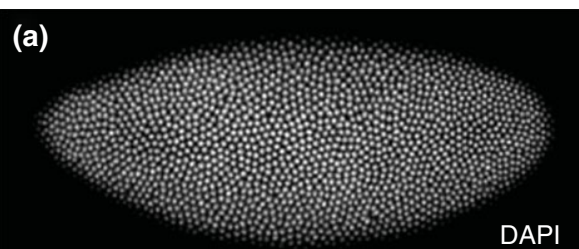

(b)
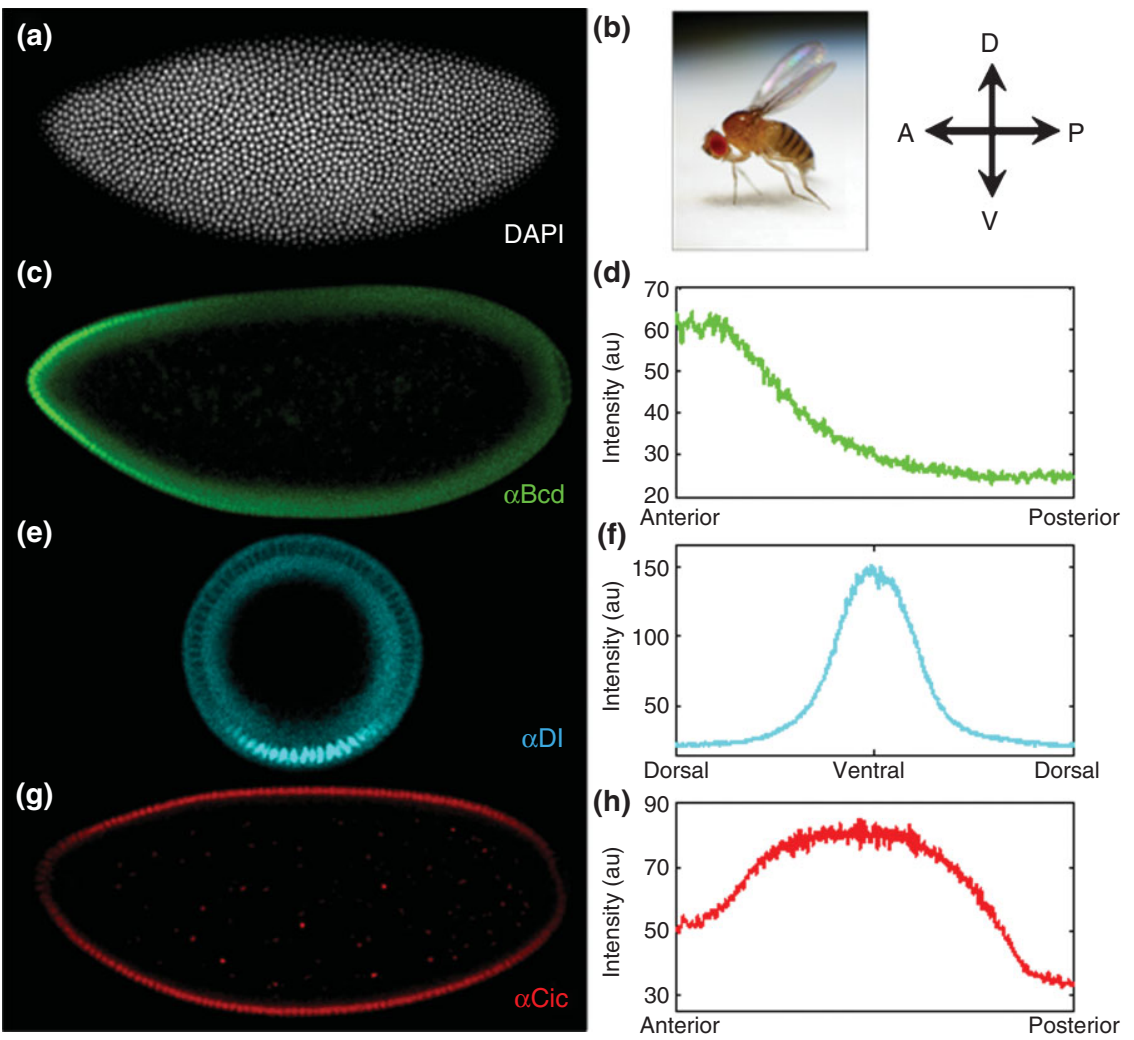

FIG URE 1 | Graded distributions of transcription factors in the early Drosophila embryo. (a) Spatially uniform arrangement of nuclei in a fixed embryo, $\sim 2 \mathrm{~h}$ after fertilization. No morphological asymmetries are apparent at this point of development. Nuclei are visualized by DAPI staining. (b) Adult Drosophila, about to take off. Schematic on the right provides the orientation of the anteroposterior (AP) and dorsoventral (DV) body axes.

(c) Concentration gradient of Bicoid (Bcd), a transcription factor that organizes anterior development of the embryo. The Bcd distribution is visualized by antibody staining in a fixed embryo. (d) Fluorescence intensity profile of the signal from an embryo stained with a-Bcd antibody. (e) Nuclear localization gradient of Dorsal (DI), a transcription factor that organizes DV patterning of the embryo. In this image, the nuclear distribution of DI is visualized in a vertically oriented embryo. (f) Quantified fluorescence intensity of nuclear Dl, based on a-Dl antibody staining. (g) Fixed embryo stained with an antibody that recognizes Capicua (Cic), a transcriptional repressor downregulated at the terminal regions of the embryo. (h) The graded pattern of Cic concentration has minima at both anterior and posterior poles.

is translated into a protein that generates a longrange concentration profile. The gradients that control dorsoventral (DV) and terminal patterning arise through a different mechanism, which involves localized activation of receptors on the surface of the embryo. In both cases, the spatially uniform receptors are activated by ligands generated in a spatial pattern that is also predefined during egg development. Thus, early development of the Drosophila embryo provides a clear example of multiple concurrent inductive events.

Studies of pattern formation in the early Drosophila embryo provide insights into general principles of inductive patterning mechanisms. For example, some aspects of pattern formation by the Dl gradient are essentially identical to those discovered during pattern formation in the vertebrate neural tube..$^{15,16}$ Over the past decade, analyses of pattern formation in the Drosophila embryo and other systems that depend on morphogens have begun to undergo a transformation to a stage in which inductive signals and their effects are studied at a quantitative level. ${ }^{17-29}$ The results of these studies enable the formulation of mathematical models that can be used to organize existing results and lead to a deeper understanding of pattern formation mechanisms. ${ }^{30-40}$

Our review provides an introduction to the basic mathematical models of gradient formation and interpretation, using the Drosophila embryo as the main example. When studying these models, the reader should keep in mind a quote from Turing ${ }^{5}$ : '. . a mathematical model of the growing embryo will be described. This model will be a simplification and an idealization, and consequently a falsification. It is to be hoped that the features retained for discussion are those of greatest importance in the present state of knowledge.' Over the five decades since these words were written, the state of knowledge has changed 


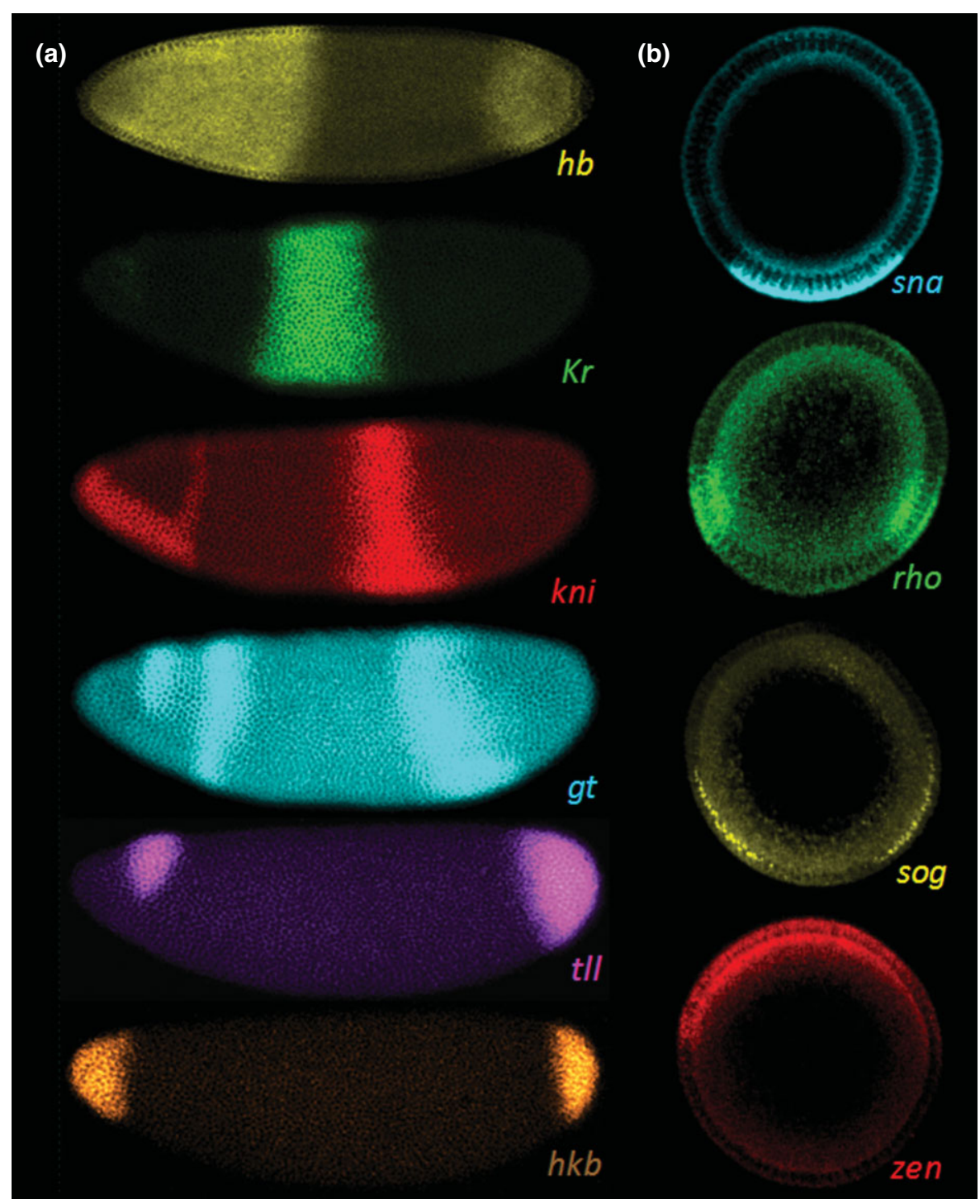

FIG URE 2 | Fluorescent in situ hybridization images showing the expression patterns of some of the genes regulated by Bicoid (Bcd), Capicua (Cic) and Dorsal (DI). (a) Expression domains of Bcd and Cic targets along the anteroposterior (AP) axis of the embryo. Note that hunchback ( $h b$; yellow), Krüppel (Kr; green), knirps (kni; red), giant (gt; blue), tailless (tll; magenta) and huckebein ( $h k b$; orange) are expressed in distinct domains along the AP axis. In anterior region of the embryo, the expression domains of these genes dependend on the activating functions of the Bcd gradient and the repressive functions of Cic. Anterior: left. (b) Expression domains of DI targets. snail (sna; blue) is only detected in the ventral regions of the embryo, while rhomboid (rho; green) and short gastrulation (sog; yellow) are expressed in more lateral domains and zerknüllt (zen; red) is confined to the dorsal region. Shown are sections through the embryo, ventral: bottom, dorsal: top.

dramatically. With this has come a change in the status of mathematical models, which can be tested now using an ever increasing number of experimental techniques. However, striking a balance between the realism of mathematical models and their practical value remains an art. As a rule, it is best to start with the simplest possible model, which captures the essence of observed patterns and can be used to do a back-of-the-envelope calculation. This is the approach we take in our review, which is addressed to students of developmental and quantitative biology.

\section{FORMATION OF MORPHOGEN GRADIENTS}

One of the simplest mechanisms for morphogen gradient formation relies on the diffusion and degradation of locally produced chemical signals. ${ }^{4,41,42}$ This mechanism can operate in both intracellular and extracellular compartments, establishing chemical gradients with characteristic length scales ranging from microns to millimetres. Importantly, each of the three ingredients of this mechanism can be realized in 
(a) Bcd Cic

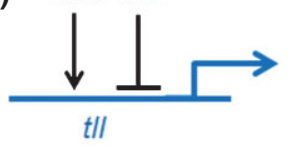

(b)

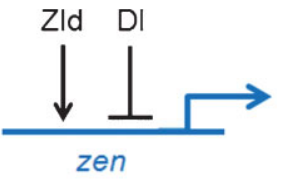

(c)

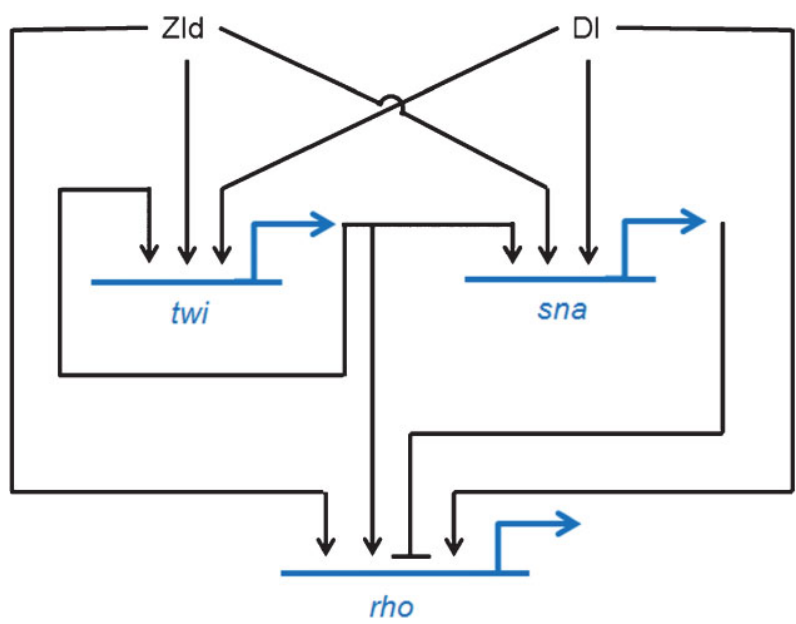

FIG URE 3 | Fragments of regulatory networks that convert maternal morphogen gradients into patterns of zygotic gene expression. (a) Anterior expression pattern of tailless (tll) depends on its direct activation by Bicoid (Bcd) and repression by Capicua (Cic). (b) Dorsal (DI) expression of zerknüllt (zen) is generated by a combination of graded DI, which acts a direct repressor, and spatially uniform Zelda (Zld), an early activator of zygotic transcription. Dl-dependent repression of zen also depends on Cic (not shown). (c) Lateral stripes of rhomboid ( $r h o$ ) expression along the dorsoventral axis of the embryo are generated by a network that combines a coherent feedforward loop, an incoherent feedforward loop, and a positive feedback autoregulation motif. DI regulates rho both directly and through Twist (twi, an activator) and Snail (sna, a repressor). In addition to sna and rho, Twi also regulates its own expression.

multiple ways. Signal production can be mediated by localized protein synthesis, secretion, or phosphorylation. Degradation can be realized by proteolysis or receptor-mediated endocytosis. Diffusion can result from any form of nondirected transport, such as molecular diffusion or repeated rounds of ligand internalization and recycling.

In most examined experimental systems to date, the basic gradient mechanism is augmented by multiple additional effects, such as directed transport or complex chemical interactions. However, a relatively simple class of linear reaction-diffusion models frequently provides a very insightful first step in the analysis of biological systems. Below we review two essential characteristics of gradient formation predicted by these models and illustrate how they can be used to address some of the key questions arising in studies of morphogen gradients. First, it is important to determine what controls the length scale of the gradient. Second, it is important to establish whether gradients reach steady states on time scales relevant for developmental patterning. Given a mathematical model of gradient formation, answers to these questions can be obtained by evaluating an analytical expression for a quantity that we call the local accumulation time, defined below.

\section{Steady State Gradient and Definition of Local Accumulation Time}

Much model-based analysis of morphogen gradients requires access to time-dependent solutions of the underlying model equations. For most existing models, time-dependent solutions can be obtained only numerically. However, given the high uncertainty in model parameters, detailed numerical solutions are not always useful. Instead, it is more practical to have simple analytical expressions that depend explicitly on model parameters (to analyze the effects of their variations) and can be used for back-of-theenvelope calculations. We now outline the approach that leads to such analytical expressions based on the steady state solution of the model, and give a definition for the local accumulation time, the latter of which provides a compact summary of concentration dynamics at a given position within the patterned field. The approach we present can be readily applied to mathematical models that describe different forms of signal release, tissues of different sizes, and cascades of reaction-diffusion processes. ${ }^{43-45}$

We focus our discussion on models formulated in one spatial dimension and assume that signal production starts at time zero, when morphogen concentration is zero throughout the system (Figure 4). At long times, the time-dependent concentration profile approaches a steady state that is determined by the balance of localized synthesis, diffusion, and degradation. The steady state concentration field is denoted by $C_{s}(x)$, where $x$ is the spatial coordinate within the tissue. In the rest of this presentation, we will assume that, for all points within the patterned domain, the morphogen concentration approaches its steady state monotonically, i.e., without overshoots. These cases are clearly important, but we will neglect them for now.

Cells (or nuclei) at different positions within the tissue will reach their approximate steady state concentrations on different time scales, which depend on the parameters of the problem and on the distance from the morphogen source (Figure 4(c)). As a first step in dealing with these dynamics, let us consider the following function, which can be interpreted as the fractional deviation of the concentration from its 
(a)

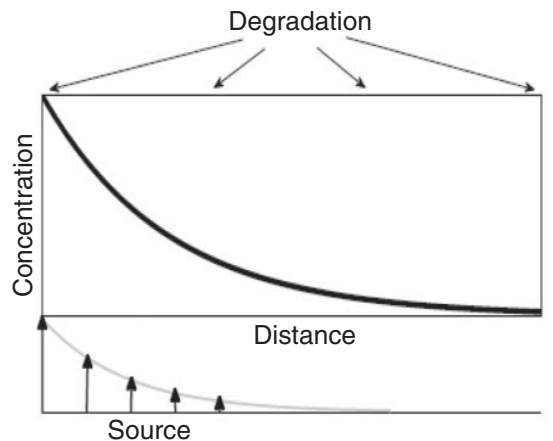

(c)

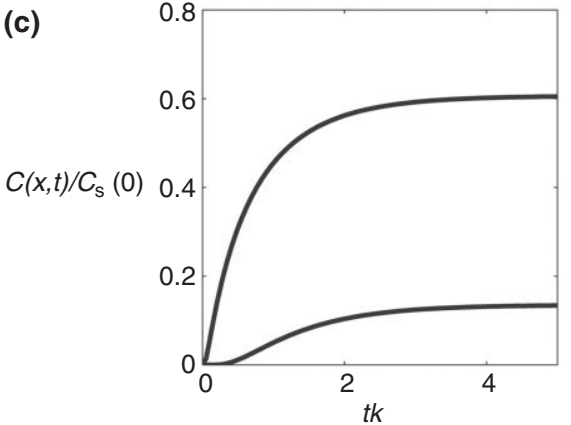

(b)

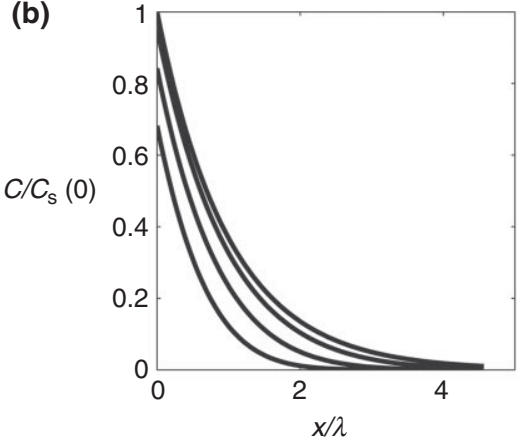

(d)

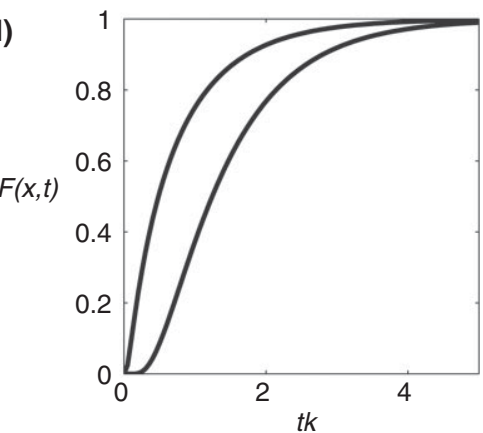

FIG URE 4 | Local accumulation time. (a) Schematic of morphogen gradient formation by synthesis, diffusion, and spatially uniform degradation. The source of morphogen production is distributed exponentially. (b) Concentration profile at four different times: $t=1 / 2 k, 1 / k, 2 / k, \infty$. The concentration profile has been plotted for $\lambda_{s}=0$, and scaled by the maximal concentration at $x=0, C_{s}(0)=Q / \sqrt{D k}$. The spatial coordinate has been rescaled by the dynamic length scale, $\lambda=\sqrt{D / k}$. (c) Concentration dynamics at $x=\lambda / 2$ and $x=2 \lambda$. (d) Local kinetics of the fractional deviation from the steady state concentration, plotted at two different positions, $x=\lambda / 2$ and $x=2 \lambda$.

steady value at a given time (Figure 4(d)):

$$
F(x, t)=1-\frac{C(x, t)}{C_{s}(x)} .
$$

Clearly, $1-F(x, t)=C(x, t) / C_{s}(x)$ is the fraction of the steady state concentration that has been accumulated at position $x$ by time $t$. Furthermore, the fraction of the steady state value accumulated between times $t$ and $t+d t$ is equal to $F(x, t)-F(x, t+d t)$. As the difference between these two times tends to zero, $F(x, t)-F(x, t+d t)$ can be approximated by a time derivative:

$$
F(x, t)-F(x, t+d t) \approx-\frac{\partial F(x, t)}{\partial t} d t
$$

Hence, $-\left[\frac{\partial F(x, t)}{\partial t}\right] d t$ can be interpreted as the fraction of the steady state concentration accumulated between times $t$ and $t+d t$. We can use this to define the local accumulation time, $\tau(x)$, as the mean time needed to form a steady gradient at a given point in space. In this definition, we add the contributions of all time intervals from zero to infinity, weighed by the corresponding fraction of the steady state concentration that has been accumulated during this interval. The result is the following integral ${ }^{43}$ :

$$
\tau(x)=\int_{0}^{\infty} t\left[-\frac{\partial F(x, t)}{\partial t}\right] d t .
$$

Importantly, this integral can be evaluated without knowledge of the time-dependent concentration profile. Details of relevant computations are described in recent papers, which provide expressions for $\tau(x)$ for several canonical versions of the source-diffusion-degradation (SDD) model. ${ }^{43-45}$

Possible configurations of a regulatory region with two binding sites for activator A (oval) and B (pentagon).

\section{Using the Local Accumulation Time to Analyze Gradient Dynamics}

Calculation of $\tau(x)$ can be used to decide whether the gradient reaches a steady state on a time scale relevant for developmental patterning. To do this, $\tau(x)$ must be compared to the time allotted for a specific developmental event. We denote this time by $T_{\mathrm{dev}}$. For instance, the time by which the Bcd gradient must be established, in order to perform its patterning 
function, is less than 90 minutes, from the onset of Bcd synthesis to cellularization. ${ }^{46}$

When $\tau(x)$ is significantly less than $T_{\mathrm{dev}}$ for all $x$ (as a rule of thumb, one can use $\tau(x)<T_{\mathrm{dev}} / 3$ ), the tissue is patterned by a steady state gradient. The length scale of this gradient can be extracted from the steady state concentration profile, given by $C_{s}(x)$. On the other hand, when $\tau(x)$ is not very different from $T_{\text {dev }}$ or exceeds it, the tissue is patterned by a time-dependent signal.

The length scale of the gradient at a given time can be extracted from an approximate time-dependent solution, which can be constructed based only on the steady profile and the local relaxation time. To construct this approximation, the approach to steady state at a given point is approximated by a single exponential, with the decay time equal to $\tau(x)$ :

$$
C_{\text {approx }}(x, t)=C_{s}(x) \times(1-\exp (-t / \tau(x))) .
$$

Clearly, the exact and approximate solutions match both when $t=0$ and when $t=\infty$. The approximation also works quite well at intermediate times that are not much smaller than $\tau(x) .{ }^{43}$ Importantly, this approximation allows us to get around the problem of finding the exact expression for the time-dependent solution. Up until this point, we have not specified the model or its parameters. We do this in the next section, for a commonly used and experimentally validated model of the Bcd gradient. First, we provide the analytical expressions for $C_{s}(x)$ and $\tau(x)$ and then supplement these expressions with numerical estimates of model parameters.

\section{Steady State and Local Accumulation Time in a Model of the Bcd Gradient}

Existing models of $\mathrm{Bcd}$ gradient formation have different levels of complexity. ${ }^{47}$ All of them account for the localized synthesis of Bcd protein in the anterior region of the embryo, together with diffusion, and degradation. A commonly used model takes the form of the following reaction-diffusion problem (a recent review provides a good introduction to reaction-diffusion models ${ }^{4}$ ):

$$
\begin{aligned}
\frac{\partial C(x, t)}{\partial t} & =D \frac{\partial^{2} C(x, t)}{\partial x^{2}}-k C(x, t)+Q(x) \\
D \frac{\partial C(0, t)}{\partial x} & =0, D \frac{\partial C(L, t)}{\partial x}=0 \\
C(x, 0) & =0 .
\end{aligned}
$$

$C(x, t)$ represents the time-dependent concentration of $\mathrm{Bcd}$ protein, $t$ is the time from the onset of
Bcd synthesis; $0<x<L$ is the distance from the anterior pole of the embryo with the size of the embryo denoted by $L$. The first of these equations accounts for the rate of change of Bcd concentration over time, with contributions from diffusion of the Bcd protein, first order degradation, and spatially distributed synthesis. The spatial distribution of Bcd synthesis is approximated by a decaying exponential function: $Q(x)=\left(Q_{0} / \lambda_{s}\right) \exp \left(-x / \lambda_{s}\right)$, which represents the gradient of $b c d$ mRNA. ${ }^{46}$ The second and third equations are the no-flux boundary conditions, reflecting the fact that the Bcd protein cannot leave the embryo. The last equation states that the protein concentration at $t=0$ is equal to zero.

This model has only a handful of parameters: the size of the system $(L)$, the signal diffusivity and the degradation rate constant ( $D$ and $k$, respectively), and two parameters that characterize the pattern of signal production $\left(Q_{0}\right.$ and $\left.\lambda_{s}\right)$. The experimentally observed gradient decays to zero before the posterior pole ${ }^{17}$ $(x=L)$, which implies that the model operates in a regime where the solution is independent of the size of the system. This reduces the number of parameters to only four. Furthermore, when Bcd synthesis is localized right at the anterior pole (corresponding to $\lambda_{s} \approx 0$, an assumption that will be relaxed in the next section), the steady is given by a single decaying exponential:

$$
C_{s}(x)=\frac{Q_{0}}{D \lambda} \exp (-x / \lambda)
$$

where $\lambda=\sqrt{D / k}$ is the dynamic length scale, and can be interpreted as the average distance to which the signal molecule diffuses before it is degraded.

One can show that local accumulation time is a linear function position ${ }^{43}$ :

$$
\tau(x)=\frac{1}{2 k}\left(1+\frac{x}{\lambda}\right) .
$$

Unlike the steady state profile, the local accumulation time does not depend on the strength of the source of morphogen production. This is always true for linear degradation models.

\section{Combining Analytical Results with Numerical Estimates of Model Parameters}

In order to investigate the implications of our calculations upon the Bcd gradient, we will now combine the expressions for the steady state profile and local accumulation time with numerical values of Bcd diffusivity, lifetime, and the spatial pattern of synthesis. We will use $D \approx 4 \mu \mathrm{m}^{2} / \mathrm{s}$, an estimate 
based on a combination of fluorescence recovery after photobleaching (FRAP) and fluorescence correlation spectroscopy (FCS) studies with a eGFP-tagged Bcd protein. ${ }^{18}$ The lifetime of the $\mathrm{Bcd}$ protein, equal to $1 / k$ for the first order degradation kinetics assumed here, is $\approx 50$ minutes, based on optical pulse-chase experiments with a photoswitchable version of the fluorescently tagged Bcd protein. ${ }^{48}$

With these choices of $D$ and $k$, we get $\lambda \approx$ $110 \mu \mathrm{m}$, which leads to the spatial profile of $\tau(x)$ shown in Figure 5(a). The minimal value of $\tau(x)$ is 33 minutes, reached at $x=0$ (the 'anterior pole of the embryo'). The most distant gene known to be directly controlled by Bcd is located two thirds along the embryo, at $x \approx 375 \mu \mathrm{m} .{ }^{12}$ At this location, $\tau(x)=110$ minutes. Comparing these numbers to 90 minutes, the upper limit of the time of the Bcd gradient formation, we see that, for a significant fraction of the anteroposterior (AP) axis, concentrations do not reach their steady values. In this regime, the spatial profile of $\mathrm{Bcd}$ can be estimated using the analytical expression

(a)

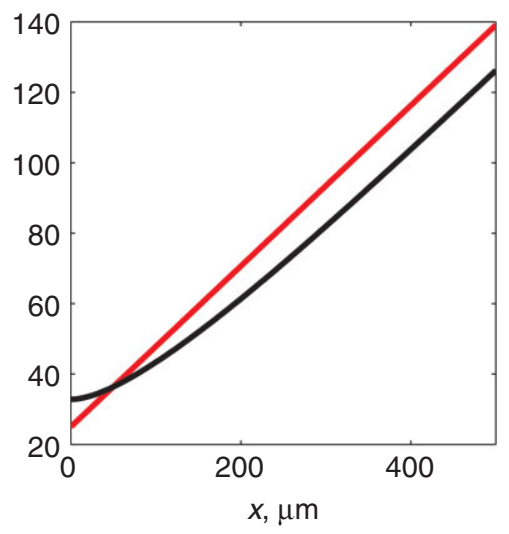

(b)

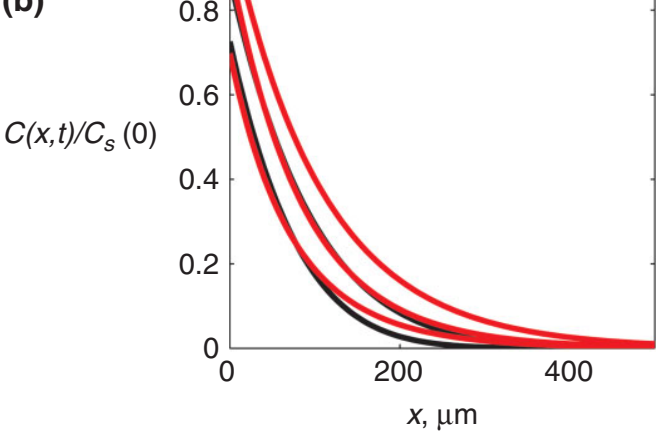

FIGURE 5 | Dynamics in the model of Bicoid (Bcd) gradient formation. (a) Local accumulation time as a function of position, calculated for $D=4 \mu \mathrm{m}^{2} / \mathrm{s}, 1 / \mathrm{k}=50 \mathrm{~min}, \lambda_{s}=0 \mu \mathrm{m}$ (black) and $\lambda_{s}=50 \mu \mathrm{m}$ (red). (b) Morphogen concentration profiles, evaluated at $30 \mathrm{~min}, 60 \mathrm{~min}$, and at steady state, based on exact and approximate solutions (black and red curves, respectively). for $C_{\text {approx }}(x, t)$, and it is remarkably close to the exact profile, $C(x, t)$ (Figure $5(\mathrm{~b})$ ).

We now ask what happens when the spatial pattern of Bcd synthesis is itself distributed in a gradient, as suggested by the quantitative analysis of $b c d$ mRNA? In this case (where $\lambda_{s}>0$ ), an expression for the local accumulation time can also be derived:

$$
\begin{aligned}
\tau(x)=\frac{1}{2 k}[ & \left(1+\frac{x}{\lambda}\right) \frac{\lambda \exp (-x / \lambda)}{\lambda \exp (-x / \lambda)-\lambda_{s} \exp \left(-x / \lambda_{s}\right)} \\
& \left.+\frac{2 \lambda_{s}^{2}}{\lambda_{s}^{2}-\lambda^{2}}\right] .
\end{aligned}
$$

To evaluate this expression, we need $\lambda_{s}$, a measure of the spatial extent of signal production. Direct measurements of the spatial pattern of $\mathrm{Bcd}$ protein synthesis are not available. As a proxy for this pattern, we can use the results of fluorescence in situ hybridization (FISH) experiments that measured the distribution of $b c d$ mRNA. ${ }^{46}$ Quantitative analysis $b c d$ FISH data reveals that $90 \%$ of $b c d$ RNA particles are located in the anterior tenth of the embryo. Based on these measurements, $\lambda_{s} \approx 50 \mu \mathrm{m}$. Combining this number with the previous estimates of $\lambda=110 \mu \mathrm{m}$ and $1 / k=50$ minutes, we get the red curve in Figure $5(\mathrm{a})$. We see that the effect of varying $\lambda_{s}$ is quite modest. We stress that these conclusions are based on parameters that have been estimated very recently and are yet to be confirmed by independent measurements and experimental techniques.

\section{Signal Transduction}

Formation of the morphogen gradients that control DV and terminal patterning of the Drosophila embryo is considerably more complex than formation of the Bcd gradient. In both of these systems, pattern formation is initiated by locally produced extracellular ligands that activate uniformly expressed plasma membrane receptors. The ligand-bound receptor triggers a signal transduction cascade that changes the abundance, phosphorylation state, or nuclear localization of a transcription factor, which thereby acts as a spatial regulator of gene expression. Thus, pattern formation is based on the localized activation of receptor-dependent signaling cascade.

For the DV patterning system, the receptor is Toll, which signals through the NF- $\kappa$ b pathway and culminates in nuclear accumulation of Dl. Toll receptor signaling is involved in numerous aspects of cellular responses across species, but its tissue patterning function appears to be restricted to insects. For the terminal system, the receptor is Torso, a 
tyrosine kinase, which signals through a highly conserved MAPK signaling cassette. Graded activation of receptor tyrosine kinases and MAPK is used ubiquitously throughout the development of multiple species. Below, we provide a very brief overview of the steps between localized activation of Torso to the generation of a gradient of transcriptional activity that organizes terminal patterning of the embryo. ${ }^{49}$

The spatial pattern of Torso occupancy depends on the localized production of its ligand Trunk, which is generated in its active form only at the embryonic poles. ${ }^{50}$ Active ligand diffuses in the extracellular space above the plasma membrane. Ligand diffusion is interrupted by its binding to Torso, activating the enzymatic activity of this receptor. Active receptor is internalized, thus terminating the process of ligand diffusion. Lateral mobility of Torso along the plasma membrane is very low. ${ }^{51}$ Thus, Torso plays a dual role in the formation of the terminal gradient: it transduces the signal provided by the extracellular ligand and limits its spatial range. ${ }^{52,53}$ This situation is commonly encountered in the formation of morphogen gradients in cellular tissues. ${ }^{54}$

A large number of experimentally characterized patterns that depend on locally produced extracellular ligands can be described by models based on two-state reaction-diffusion systems, where a diffusible particle can be in two states: diffusible or immobile. ${ }^{55}$ In the terminal system, the diffusible particle corresponds to free active Trunk and the immobile particle corresponds to Trunk bound to Torso. In general, the degradation rate constants in the two different states are different. For example, in the terminal system, it is believed that ligand is degraded only in the immobile state (when bound to Torso). Detailed analysis of gradient formation in two-state reaction-diffusion systems and cascades of such systems is described in a number of recent papers. ${ }^{45,55,56}$

A key step in the terminal patterning process is the formation of the two-peaked pattern of activity pattern of MAPK. MAPK phosphorylates several intracellular substrates, including Cic, which is essential for the terminal patterning of the embryo. In the absence of phosphorylation by MAPK, Cic represses gene expression throughout the embryo. Two of the genes repressed by Cic are tailless $(t l l)$ and huckebein $(h k b)$. In response to phosphorylation by MAPK, Cic is degraded. The Cic degradation pattern is graded and negatively correlated with the pattern of MAPK activation. ${ }^{57}$ This graded pattern gives rise to the localized derepression of $t l l$ and $h k b$. Thus, similar to the system that forms the Bcd gradient, the result of Torso signaling is graded distribution of a transcription factor. In the rest of the review, we discuss models that can be used to describe how a graded distribution of transcription factors is converted into spatial patterns of gene expression.

\section{INTERPRETATION OF MORPHOGEN GRADIENTS}

\section{Local Regulation of Gene Expression}

A key issue in the context of morphogen-dependent patterning is to understand how and why different genes are expressed in different positions, corresponding to different concentrations of a morphogen. ${ }^{3}$ As a rule, the expression of a single gene is regulated by multiple transcription factors. Thus, to understand how morphogens control gene expression, we need to consider the joint effects of multiple regulators, which may be either dependent or independent on the morphogen gradient.

To simplify things, we will assume that the expression rate of a given gene, i.e., the number of new transcripts generated per unit time, at a given position within the tissue, can be written as an algebraic function of local concentrations of transcription factors. The form of this function depends on the regulatory region of the gene. Amongst other things, it depends on the binding sites of specific transcription factors and the distances between these sites.

Morphogen gradients can control gene expression both directly and indirectly, through transcriptional cascades. An archetypal example of a transcriptional cascade is regulation of the gap and pair rule genes during Drosophila segmentation. Bcd directly regulates expression of the gap genes, in the form of broad domains; the products of these genes work with Bcd to establish striped expression of the pair rule genes. ${ }^{58}$

The expression levels of both direct and indirect targets of a morphogen are affected by quantitative manipulations of the morphogen level. The strength of the effect depends on the number and strength of the binding sites for the morphogen within the regulatory sequences of its specific targets. However, this is only part of the story, because each step within such a cascade can be regulated by additional, auxiliary factors that do not depend on the graded signal. These factors can be either spatially uniform or graded. For instance, gene expression along the DV axis depends both on Dl, which is graded, and Zelda (Zld), a uniformly distributed transcriptional activator. ${ }^{59,60}$

\section{Direct Effects of Morphogen Gradients}

Some of the key aspects of gene regulation by a combination of graded and uniform signals can 
(a)

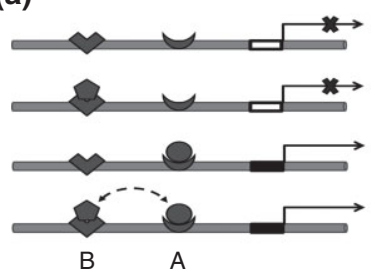

(c)

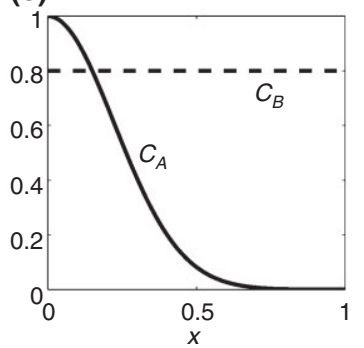

(b)

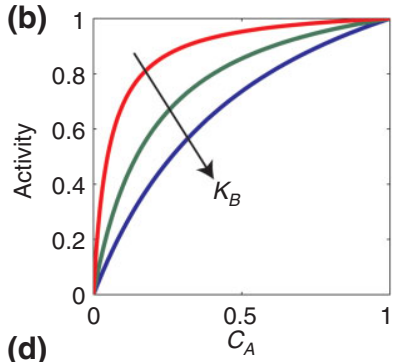

(d)

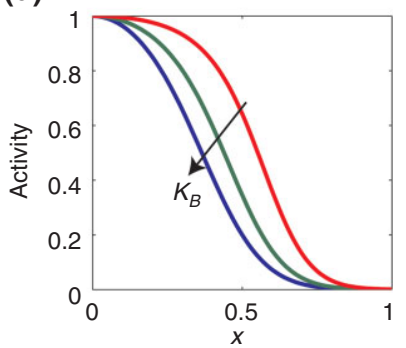

FIGURE 6 | Gene regulation by combination of graded and uniform signals. (a) Possible configurations of a regulatory region with two binding sites for activator A (oval) and B (pentagon). (b) Input/output map of the regulatory region as a function of concentration of factor $A$, at different concentrations of factor $B$, or at different values of the cooperativity parameter (see text for details). Model parameters are as follows (see text for details): $K_{A}=1, K_{B}=8,4,200$, and $\omega=200$. (c) Spatial distributions of two transcription factors. (d) Normalized spatial pattern of the activity of the regulatory region.

be illustrated by the following model, which can be viewed as a simplified description of the first step within a transcriptional cascade. The model assumes that the expression rate of a gene depends on the probability of finding its regulatory region bound by a specific complement of transcription factors. This type of mathematical description of transcriptional regulator is called a site occupancy model. ${ }^{28,34,61-63}$

Consider a gene controlled by two factors, A and $\mathrm{B}$, each of which has a single binding site within a hypothetical regulatory region (Figure 6(a)). The concentrations of these factors are denoted by $C_{A}$ and $C_{B}$. Let us think of $C_{A}$ as the graded concentration of a morphogen; $C_{B}$ reflects the concentration of auxiliary signal and it can be either uniform or graded.

The regulatory region can be found in four different states: one state with both sites empty, two states with a single site occupied, and one state with both sites bound by their corresponding transcription factors. We assume that binding of the morphogen is a necessary condition for activation of the regulatory region. This can be realized in two different ways, when A is bound, either by itself or together with B. When the binding reactions are in equilibrium, the probability of finding the regulatory region in either one of the states necessary for gene activation is

given by

$$
\begin{aligned}
P\{A \text { bound }\} & =P\{A\}+P\{A \& B\} \\
& =\frac{C_{A}+C_{A} C_{B} \omega / K_{B}}{K_{A}+C_{A}+K_{A} C_{B} / K_{B}+C_{A} C_{B} \omega / K_{B}}
\end{aligned}
$$

In this expression, $K_{A}$ and $K_{B}$ are the corresponding equilibrium-binding constants, which characterize the binding affinities of $\mathrm{A}$ and $\mathrm{B}$ to their respective sites, when taken in isolation. $\omega$ is a measure of cooperativity, which reflects the ability of factor $\mathrm{B}$ to change the binding affinity of $\mathrm{A}$ to its site. Clearly, when $\omega=1, P\{A$ bound $\}=C_{A} /\left(K_{A}+C_{A}\right)$, which corresponds to the case of no cooperativity. When $\omega>1$, the auxiliary factor B can be viewed as a coactivator that 'recruits' factor A to its binding site.

Let us consider the implications of this model for the spatial control of gene expression when $A$ is graded and $B$ is uniform. To make things specific, the concentration field of $C_{A}$ is described by a single exponential (Figure 6(b)), which could be established by processes of reaction and diffusion. A representative pattern of transcriptional activity is shown in Figure 6(c). According to the model, this pattern is affected by changes in the level of the morphogen $\left(C_{A}\right)$ as well as by changes in the strength of its binding site $\left(K_{A}\right)$. Note that the spatial pattern of transcriptional activity can also be affected by the uniform factor, through changes in $C_{B}, K_{B}$, and $\omega$. Predictions of the model can be used to interpret the results of recent experiments, which demonstrated that uniform factors play an important role in gene regulation, by morphogen gradients.

Our illustrative model can be generalized to the case when factor $\mathrm{B}$ is a repressor and/or when its spatial distribution is non-uniform. The main conclusion from analyzing of such models is that gene regulation by a morphogen can be tuned in a variety of ways by combinatorial interactions. As a consequence, several alternative models can explain the fact that different genes are expressed in different patterns. Observed variation in expression patterns can stem from differences in the number or strength of binding factors for the morphogen itself. Alternatively, they can reflect differential response to auxiliary signals. This point has been illustrated by recent studies of gene regulation by the $\mathrm{Bcd}$ gradient. ${ }^{64,65}$

\section{Dynamic Models of Gene Expression}

Before moving on to transcriptional cascades, we need to set-up a simple dynamic model that describes 
the expression level of one direct target of a spatially graded signal. This model provides a connection between the dynamics of patterning signals and spatiotemporal patterns of gene and protein expression. Solution of this model will be used to discuss pattern formation by a combination of direct and indirect effects. We use perhaps the simplest possible dynamic model of transcriptional regulation. This model has only two variables, denoted by $C_{m}$ and $C_{p}$, which correspond to the concentration of mRNA and protein, respectively, of a gene controlled by a graded chemical signal.

Most importantly, production of mRNA is determined by the state of a gene regulatory region. In the simplest case, it is proportional to the probability of finding this region in a state favorable for transcription. For illustrative purposes, we will use a model with a single site for an activator, whose timedependent concentration is denoted by $C_{A}(x, t)$ and can be found from the models of morphogen gradient formation. Protein production rate is proportional to the amount of mRNA. The degradation rates for both mRNA and protein are assumed to follow first order kinetics. With these assumptions, the model takes the following form:

$$
\begin{aligned}
\frac{d C_{m}}{d t} & =G_{1} \frac{C_{A}}{K_{A}+C_{A}}-k_{\mathrm{deg}}^{m} C_{m} \\
\frac{d C_{p}}{d t} & =G_{2} C_{m}-k_{\mathrm{deg}}^{p} C_{p} .
\end{aligned}
$$

In these equations, $G_{1}$ and $G_{2}$ are the proportionality constants in the simplified descriptions of transcription and translation, respectively, and $K_{A}$ is the affinity of a binding site for an activator. If the rate constant for transcript degradation $\left(k_{\mathrm{deg}}^{m}\right)$ greatly exceeds the rate constant for protein degradation $\left(k_{\mathrm{deg}}^{p}\right)$, we can use a steady state approximation for the transcript level, which leads to a single variable model for protein concentration:

$$
\frac{d C_{p}}{d t}=\frac{G_{2} G_{1}}{k_{\mathrm{deg}}^{m}} \frac{C_{A}}{K_{A}+C_{A}}-k_{\mathrm{deg}}^{p} C_{p} .
$$

Because morphogen concentration, $C_{A}$, is a function of space and time, solution of this equation will depend on both $x$ and $t$. In the rest of this section, we limit our discussion to steady state solutions, which correspond to the case when the time scale of protein degradation is faster than the time scale of morphogen gradient formation. This leads to the following expression for the spatial distribution of protein product of a gene regulated by a morphogen gradient:

$$
\begin{aligned}
C_{p}(x, t) & \approx \frac{G_{2} G_{1}}{k_{\mathrm{deg}}^{m} k_{\mathrm{deg}}^{p}} \frac{C_{A}(x, t)}{K_{A}+C_{A}(x, t)} \\
& =C_{p}^{\max } \frac{C_{A}(x, t)}{K_{A}+C_{A}(x, t)} .
\end{aligned}
$$

Here $C_{p}^{\max } \equiv G_{2} G_{1} /\left(k_{\mathrm{deg}}^{m} k_{\mathrm{deg}}^{p}\right)$ is the maximal possible protein concentration, which is attained when activator concentration greatly exceeds the equilibrium-binding constant $\left(C_{A} K_{A}\right)$. In this regime, $C_{p}(x, t)$ is directly proportional to the probability of finding the gene regulatory region in a state favorable for transcription. Under the assumptions above, the same will hold true for models of more complex regulatory regions, e.g., those controlled by multiple activators and repressors.

\section{Formation of Nonmonotonic Patterns by Incoherent Feedforward Loops}

One of the simplest and most commonly encountered motifs in transcriptional cascades is an incoherent feedforward loop, a three-node network where an input $(\mathrm{A})$ activates a gene $(\mathrm{B})$ and its repressor $(\mathrm{C}){ }^{66}$ This network can convert a morphogen gradient into a spatially nonmonotonic pattern of gene expression. To illustrate this point, let us consider a hypothetical gene regulatory region with two binding sites: one for (a)
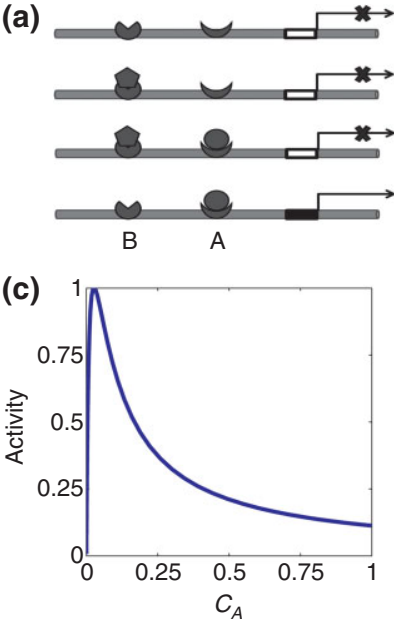

(b)

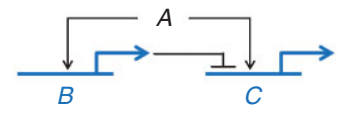

(d)

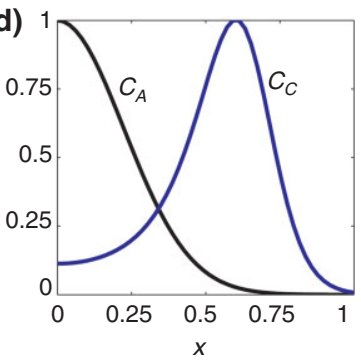

FIG URE 7 | Spatial signal processing by the incoherent feedforward loop. (a) Possible configurations of a regulatory region controlled by an activator and repressor. (b) Structure of an incoherent feedforward loop. (c) Normalized activity of the regulatory region as a function of morphogen concentration. Model parameters are as follows (see text for details): $K_{A}^{C}=0.05, K_{B}^{C}=0.01, K_{A}^{B}=50, C_{B}^{\max }=35$. (d) Normalized spatial distribution of the morphogen (black) and normalized spatial distribution of the activity of the regulatory region of gene $C$ (blue). 
an activator, distributed in a gradient, and one for a repressor, which is induced by the same activator (Figure $7(\mathrm{a})$ ). We assume that this module is active when it is bound by an activator and not bound by a repressor. Furthermore, we assume that binding reactions are in equilibrium and that binding events of repressor and activator are independent. With these assumptions, the probability of finding the region in a state bound by an activator and no repressor:

$$
\begin{aligned}
P\{A \text { bound, } B \text { not bound }\} & =P\{A\}(1-P\{B\}) \\
& =\frac{C_{A}}{K_{A}^{C}+C_{A}} \frac{K_{B}^{C}}{K_{B}^{C}+C_{B}} .
\end{aligned}
$$

$K_{A}^{C}$ and $K_{B}^{C}$ are the equilibrium-binding constants for repressor and activator within the regulatory region of gene $\mathrm{C}$. As before, let us assume that the spatial distribution of $C_{A}$ is established by an independent process of morphogen gradient formation, and leads to a given profile, $C_{A}(x)$.

To evaluate the spatial profile of the activity of the gene regulatory region in our model we need to find the spatial profile of repressor. This can be done using a model from the previous subsection, in which repressor is directly activated through a regulatory region with a single binding site, whose affinity for an activator is denoted by $K_{A}^{B}$ :

$$
C_{B}(x)=C_{B}^{\max } \frac{C_{A}}{K_{A}^{B}+C_{A}} .
$$

Substituting this into the expression for $P\{A$ bound, $B$ not bound $\}$, we get a single variable function of activator concentration. Depending on the values of model parameters, this function can have a maximum. For instance, when $K_{A}^{B}$ is much larger than the maximal concentration of the activator, $C_{B}(x) \approx C_{B}^{\max } C_{A} / K_{A}^{B}$, this leads to

$$
\begin{aligned}
& P\{A \text { bound, } B \text { not bound }\} \\
& \quad=\frac{C_{A}}{K_{A}^{C}+C_{A}} \frac{K_{B}^{C}}{K_{B}^{C}+C_{B}^{\max } C_{A} / K_{A}^{B}} .
\end{aligned}
$$

This function has a maximum at $C_{A}=$ $\sqrt{K_{A}^{C} K_{B}^{C} K_{A}^{B} / C_{B}^{\max }}$. If this concentration falls within the range of the morphogen gradient, transcriptional activity of the regulatory region of gene $\mathrm{C}$ has a maximum as a function of position. A representative example is shown in Figure 7(b). Thus, an incoherent feedforward loop can convert a spatially monotonic morphogen gradient into a nonmonotonic pattern of gene expression. This mechanism is used during the
DV patterning of the embryo, when the expression of genes needed for neural development is excluded from the presumptive mesoderm region. In this case, Dl activates both neuroectoderm-specific genes, such as sog, and Sna, which represses them in the presumptive mesoderm (Figure 2(c)).

\section{Formation of Patterns with Sharp Boundaries: Cooperativity}

Regulatory regions of genes commonly have closely spaced sites for the same transcriptional regulator. For example, clusters of $\mathrm{Bcd}$ and $\mathrm{Dl}$ binding sites are a common feature of multiple genes expressed in the early fly embryo. This structural feature of gene regulatory regions can lead to highly cooperative binding of transcription factors, which can result from attractive interactions between proteins bound to adjacent sites. Below, we will use a model with two adjacent binding sites for a single factor B. When cooperative effects are strong, the probability to find this cluster is in a state with at least one molecule of B is closely approximated by the following function:

$$
P\{B \text { bound }\}=\frac{C_{B}^{2}}{K_{B}^{2}+C_{B}^{2}} \text {. }
$$

This is a particular case of a more general Hill-type nonlinearity, $f(c)=c^{n} /\left(k^{n}+c^{n}\right)$, which is commonly used in models of transcriptional regulation. This function is more switch-like than the hyperbolic dependences used in the models of the previous sections. A measure of a switch-like behavior is provided by the fold change of the input that it is needed to change the output from $10 \%$ to $90 \%$ of the maximal value. When $n=1$, this requires an 81 -fold increase of the input, almost two orders of magnitude. On the other hand, for $n=2$ the same change can be generated by only a 9-fold change in $c$. As the value of $n$ increases, turning a system from 10 to $90 \%$ 'on' requires progressively smaller fold-changes of input.

Gene regulatory regions with homotypic binding sites for cooperatively binding transcription factors can display an increase in the sharpness of the transcriptional response to morphogen gradients. A clear example of this strategy was provided by studies of Bcd-dependent regulation of hunchback $(b b) .{ }^{23}$ In a number of cases, the emerging patterns change very abruptly, sometimes over just a couple cells, a length scale which is considerably less than the length scale on which the concentration of a morphogen changes significantly. Explaining such abrupt changes solely based on a direct response to a morphogen gradient requires very high values of $n$, which is 
inconsistent with experimentally measured values that rarely exceed $n=5$. On the other hand, a mechanism based on a positive feedback loop, which relies on only modest cooperativity, can generate patterns of essentially arbitrary sharpness. ${ }^{67}$

\section{Positive Feedback Circuit: Bistability}

To illustrate the patterning function of circuits with positive feedback loops, we analyze a hypothetical gene regulatory region with three binding sites for two activators: one site for the morphogen A and two sites for factor $\mathrm{B}$, which is the protein product of the gene controlled by this region. We assume that there is no cooperativity between factors A and B. On the other hand, binding of $\mathrm{B}$ is highly cooperative and is described by a Hill function with $n=2$. If we assume that this regulatory region is active when either one of the factors is bound, the probability of finding the region in a configuration favorable for transcription is given by

$$
\begin{aligned}
P\{A \text { or } B \text { bound }\} & =1-(1-P\{A\})(1-P\{B\}) \\
& =P\{B\}+P\{A\}(1-P\{B\}) .
\end{aligned}
$$

We can use this expression to construct a dynamical model for the concentration of factor $\mathrm{B}$ (for clarity, we suppress the dependence of $C_{A}$ and $C_{B}$ on space and time)

$$
\begin{array}{r}
\frac{d C_{B}}{d t}=k_{\operatorname{deg}}^{B, p}\left[C _ { B } ^ { \operatorname { m a x } } \left(\frac{C_{B}^{2}}{K_{B}^{2}+C_{B}^{2}}+\frac{C_{A}}{K_{A}+C_{A}}\right.\right. \\
\left.\left.\left(1-\frac{C_{B}^{2}}{K_{B}^{2}+C_{B}^{2}}\right)\right)-C_{B}\right] .
\end{array}
$$

This model has two interesting properties. ${ }^{67}$ First, the steady state profile of B can change very abruptly as a function of coordinate. Second, $C_{B}$ can exhibit persistent responses to transient inputs, e.g., to morphogen concentrations with pulse-like dynamics. In other words, the system has memory. Both of these properties stem from the fact that it has multiple steady states at zero morphogen concentration. When $C_{A}=0$, the differential equation for $C_{B}$ becomes:

$$
\frac{d C_{B}}{d t}=k_{\operatorname{deg}}^{B, p}\left[C_{B}^{\max }\left(\frac{C_{B}^{2}}{K_{B}^{2}+C_{B}^{2}}\right)-C_{B}\right] .
$$

The number and stability of steady states in this equation can be determined by analyzing the intersection of sigmoidal synthesis and linear degradation terms (Figure 8). When $C_{B}^{\max }>2 K_{B}$, the (a)

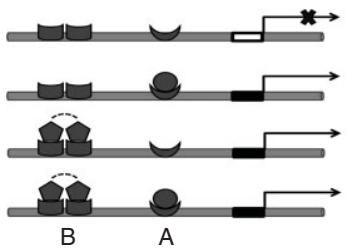

(c)

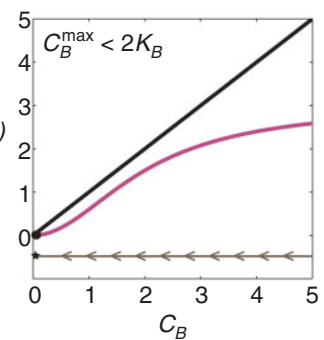

(e)

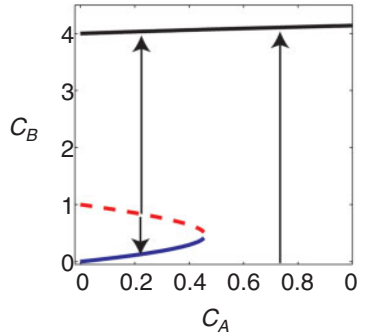

(b)

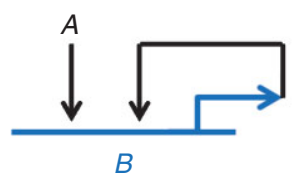

(d)

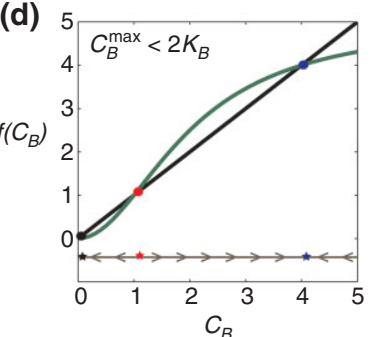

(f)

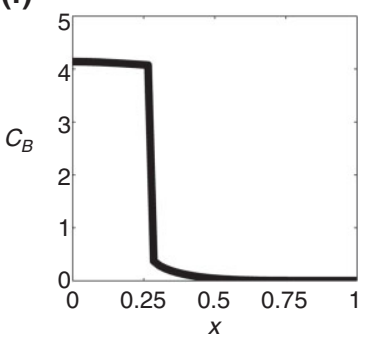

FIG URE 8 | Spatial signal processing by the positive feedback loop. (a) Possible configurations of a gene regulatory region controlled by two activators, one of which has a cluster of homotypic binding sites. (b) Structure of the positive feedback circuit. (c) Geometrical analysis of steady states and their stability at zero level of morphogen concentration. A case with a single stable steady state. Model parameters are as follows (see text for details): $C_{B}^{\max }=3, K_{A}=10$, $K_{B}=2$. (d) Geometrical analysis of steady states and their stability at zero level of morphogen concentration. A case with steady state multiplicity. Parameters are as in (c), but $C_{B}^{\max }=5$. (e) Parametric dependence of steady states on morphogen concentration $\left(C_{A}\right)$. The 'off' steady state (blue) disappears beyond critical concentration of the morphogen. Stable and unstable steady states are shown by solid and dashed curves, respectively. (f) Discontinuous spatial profile of the steady state generated by a positive feedback responding to a morphogen gradient.

curves intersect three times, which means that there are three distinct concentrations of factor B at which its synthesis and degradation balance each other. By analyzing the signs of the time derivative of $C_{B}$ at each of these concentrations, we see that the intermediate steady state is unstable (Figure $8(\mathrm{~b})$ ) and the others are stable.

Consider now what happens to each of these steady states as a function of morphogen concentration, $C_{A}$. The 'off' state exists only below a critical value of morphogen concentration, denoted by $C_{A, \text { crit }}$. At this concentration, the 'off' state collides with the intermediate unstable steady state and both them disappear. In contrast, the 'on' state remains 
stable at all morphogen concentrations, approaching $C_{B}^{\max }$ when $C_{A} K_{A}$.

This steady state picture is useful in analyzing the spatial response of an autoregulating circuit to a morphogen gradient. For simplicity, let us assume that the gradient is at steady state, given by a monotonically decaying function $C_{A, s}(x)$. If the system starts in a state with $C_{B}=0$ for $x$, then the steady state value of $C_{B}$ at a given value of $x$ depends on whether the morphogen concentration at this location is below or above the critical concentration, $C_{A, \text { crit }}$. All points that correspond to morphogen concentrations below $C_{A \text {,crit }}$ will reach the steady state that corresponds to the 'off' state of the positive feedback, in its bistable regime. At the same time, wherever $C_{A, s}(x)>C_{A \text {, crit }}$, the system will move to the steady state that is 'related' to the 'on' state at $C_{A}=0$. Because of a discontinuous change in the steady solution as a function of morphogen concentration, the steady state profile of $C_{B}$ will exhibit a discontinuity as a function of position. As a consequence the spatial distribution of $C_{B}$ will be infinitely sharp.

The same analysis can be used to understand the ability of the positive feedback system to remember transient inputs. Consider a system that has reached a steady state distribution of $C_{B}$. What happens when the morphogen concentration is now reduced to zero throughout the system? Based on analysis of the steady states with $C_{A}=0$, we see that the concentration of $C_{B}$ at all $x$ located to the right of the value that corresponds to $C_{A \text {,crit }}$ will relax back to the 'off' state $\left(C_{B}=0\right)$. On the other hand, $C_{B}$ for all $x$ to the left of this value, will change only slightly, adjusting to the 'on' state (Figure 8(c)).

Thus, a positive feedback circuit can convert a transient signal into a stable pattern of transcriptional activity. This property is essential for developmental patterning systems, which are commonly activated by transient inputs. For instance, the Bcd gradient is degraded toward the onset of cellularization and some of the gene expression patterns established by $\mathrm{Bcd}$ are maintained by mechanisms that depend on positive feedback or dual repressor circuit, which also supports bistability. ${ }^{24,68}$ Importantly, similar mechanisms have been recently shown to play essentially the same roles in vertebrate systems. ${ }^{69,70}$

\section{CONCLUSION}

We reviewed a number of simple models that can be used as building blocks in the mathematical analysis of morphogen gradients and their effects on gene expression. Biological systems necessarily use a combination of multiple strategies for the formation and interpretation of morphogen gradients. ${ }^{21,33}$ For example, understanding even the earliest steps of pattern formation by the $\mathrm{Dl}$ gradient requires simultaneous analysis of coherent and incoherent feedforward loops and positive autoregulation. ${ }^{34}$ As another example, gradient formation in the terminal system relies on a cascade of reaction-diffusion systems, the output of which is interpreted by gene regulatory regions that respond to a combination of graded and uniform signals. ${ }^{56,71}$ The differential contributions of multiple layers of regulation in such complex systems can be explored using the models reviewed in the sections above. Specific examples of this approach can be found in recent publications. ${ }^{24,25,28,37,38,63,71,72}$

We focused on patterns in one spatial dimension. However, it is clear that a combination of multiple graded signals, distributed along different axes of the tissue and acting through gene regulatory regions similar to ones discussed above can generate a variety of two-dimensional patterns. ${ }^{73,74}$ Patterns with multiple peaks, such as the striped expression patterns of the pair rule genes, can be described using models in which a single gene is controlled by multiple regulatory regions, which respond to different combinations of transcription factors. As a first approximation, these regions can be modelled independently, similar to the approaches outlined in this review. ${ }^{75}$

To summarize, the emergence of patterns of increasing complexity can be understood based on simple physicochemical models. Connecting these models to experimentally observed patterns requires a quantitative analysis of inductive signals and transcriptional responses as a function of time, space, and genetic background. Supported by quantitative experiments, mechanism-based, quantitative models provide unique insights into the robustness and evolution of developmental pattern formation processes. The early Drosophila embryo, with its simple anatomy, and a wealth of available and rapidly evolving genetic and imaging tools provides a fertile ground for testing this interdisciplinary approach.

\section{ACKNOWLEDGMENTS}

S.Y.S. thanks Alexander Berezhkovskii, Alistair Boettiger, Oliver Grimm, Johannes Jaeger, Jitendra Kanodia, Yoosik Kim, Ulrike Löhr, Dmitri Papatsenko, Michael Levine, Cyrill Muratov, John Reinitz, Christine Rushlow, 
Trudi Schüpbach, Ruth Steward, and Eric Wieschaus for helpful discussions. S.Y.S. also thanks Jitendra Kanodia, Bomyi Lim, and Yoosik Kim for invaluable help with preparing the figures.

\section{REFERENCES}

1. Wolpert L. Positional information and spatial pattern of cellular differentiation. $J$ Theor Biol 1969, 25:1-47.

2. Wolpert L. One hundred years of positional information. Trend Genet 1996, 12:359-364.

3. Ashe HL, Briscoe J. The interpretation of morphogen gradients. Development 2006, 133:385-394.

4. Wartlick O, Kicheva A, González-Gaitán M. Morphogen gradient formation. Cold Spring Harb Perspect Biol 2009, 1:a001255.

5. Turing AM. The chemical basis of morphogenesis. Phil Trans Roy Soc Lond 1952, B237:37-72.

6. Struhl G, Struhl K, Macdonald PM. The gradient morphogen Bicoid is a concentration-dependent transcriptional activator. Cell 1989, 57:1259-1273.

7. Driever W, Nusslein-Volhard C. A gradient of Bicoid protein in Drosophila embryos. Cell 1988, 54:83-93.

8. Driever W, Nusslein-Volhard C. The Bicoid protein determines position in the Drosophila embryo in a concentration-dependent manner. Cell 1988, 54:95-104.

9. Steward R. Relocalization of the Dorsal protein from the cytoplasm to the nucleus correlates with its function. Cell 1989, 59:1179-1188.

10. Roth S, Stein D, Nusslein-Volhard C. A gradient of nuclear localization of the Dorsal protein determines dorsoventral pattern in the Drosophila embryo. Cell 1989, 59:1189-1202.

11. Rushlow CA, Han KY, Manley JL, Levine M. The graded distribution of the Dorsal morphogen is initiated by selective nuclear import transport in Drosophila. Cell 1989, 59:1165-1177.

12. Porcher A, Dostatni N. The Bicoid morphogen system. Curr Biol 2010, 20:R249-R254.

13. Hong JW, Hendrix DA, Papatsenko D, Levine MS. How the Dorsal gradient works: Insights from postgenome technologies. Proc Natl Acad Sci 2008, 105:20072-20076.

14. Furriols M, Casanova J. In and out of Torso RTK signalling. EMBO J 2003, 22:1947-1952.

15. Briscoe J. Making a grade: Sonic Hedgehog signalling and the control of neural cell fate. EMBO J 2009, 28:457-465.

16. Cornell RA, Ohlen TV. Vnd/nkx, ind/gsh, and msh/ msx: conserved regulators of dorsoventral neural patterning? Curr Opin Neurobiol 2000, 10:63-71.

17. Gregor T, Wieschaus E, McGregor AP, Bialek W, Tank DW. Stability and nuclear dynamics of the Bicoid morphogen gradient. Cell 2007, 130:141-153.
18. Abu-Arish A, Porcher A, Czerwonka A, Dostatni N, Fradin C. High mobility of bicoid captured by fluorescence correlation spectroscopy: implication for the rapid establishment of its gradient. Biophys J 2010, 99:L33-35.

19. Chung K, Kim Y, Kanodia JS, Gong E, Shvartsman SY, $\mathrm{Lu} \mathrm{H}$. A microfluidic array for large-scale ordering and orientation of embryos. Nat Method 2011, 8:171-177.

20. Kanodia JS, Rikhy R, Kim Y, Lund VK, DeLotto R, Lippincott-Schwartz J, Shvartsman SY. Dynamics of the Dorsal morphogen gradient. Proc Natl Acad Sci 2009, 106:21707-21712.

21. Jaeger J, Blagov M, Kosman D, Kozlov KN, Manu, Myasnikova E, Surkova S, Vanario-Alonso CE, Samsonova M, Sharp DH, et al. Dynamical analysis of regulatory interactions in the gap gene system of Drosophila melanogaster. Genetics 2004, 167:1721-1737.

22. Jaeger J, Surkova S, Blagov M, Janssens H, Kosman D, Kozlov KN, Manu, Myasnikova E, Vanario-Alonso $\mathrm{CE}$, Samsonova M, et al. Dynamic control of positional information in the early Drosophila embryo. Nature 2004, 430:368-371.

23. Lebrecht D, Foehr M, Smith E, Lopes FJ, VanarioAlonso CE, Reinitz J, Burz DS, Hanes SD. Bicoid cooperative DNA binding is critical for embryonic patterning in Drosophila. Proc Natl Acad Sci U S A 2005, 102:13176-13181.

24. Manu, Surkova S, Spirov AV, Gursky VV, Janssens H, Kim AR, Radulescu O, Vanario-Alonso CE, Sharp DH, Samsonova M, et al. Canalization of gene expression in the Drosophila blastoderm by gap gene cross regulation. Plos Biol 2009, 7:591-603.

25. Umulis DM, Shimmi O, O'Connor MB, Othmer HG. Organism-scale modeling of early Drosophila patterning via bone morphogenetic proteins. Dev Cell 2010, 18:260-274.

26. Bollenbach T, Pantazis P, Kicheva A, Bökel C, GonzálezGaitán M, Jülicher F. Precision of the Dpp gradient. Development 2008, 135:1137-1146.

27. Kicheva A, Pantazis P, Bollenbach T, Kalaidzidis Y, Bittig T, Jülicher F, González-Gaitán M. Kinetics of morphogen gradient formation. Science 2007, 315: 521-525.

28. Parker DS, White MA, Ramos AI, Cohen BA, Barolo S. The cis-regulatory logic of Hedgehog gradient responses: key roles for gli binding affinity, competition, and cooperativity. Sci Signal 2011, 4:ra38.

29. Perry MW, Boettiger AN, Bothma JP, Levine M. Shadow enhancers foster robustness of Drosophila gastrulation. Curr Biol 2010, 20:1562-1567. 
30. Janssens H, Hou S, Jaeger J, Kim AR, Myasnikova E, Sharp D, Reinitz J. Quantitative and predictive model of transcriptional control of the Drosophila melanogaster even skipped gene. Nat Genet 2006, 38:1159-1165.

31. Perkins TJ, Jaeger J, Reinitz J, Glass L. Reverse engineering the gap gene network of Drosophila melanogaster. PLoS Comput Biol 2006, 2:e51.

32. Vakulenko S, Manu, Reinitz J, Radulescu O. Size regulation in the segmentation of Drosophila: interacting interfaces between localized domains of gene expression ensure robust spatial patterning. Phys Rev Lett 2009, 103:168102.

33. Papatsenko D. Stripe formation in the early fly embryo: principles, models, and networks. Bioessays 2009, 31:1172-1180.

34. Zinzen RP, Senger K, Levine M, Papatsenko D. Computational models for neurogenic gene expression in the Drosophila embryo. Curr Biol 2006, 16:1358-1365.

35. Ashyraliyev M, Siggens K, Janssens H, Blom J, Akam M, Jaeger J. Gene circuit analysis of the terminal gap gene huckebein. PLoS Comput Biol 2009, 5:e1000548.

36. Umulis DM, Serpe M, O'Connor MB, Othmer HG. Robust, bistable patterning of the dorsal surface of the Drosophila embryo. Proc Natl Acad Sci U S A 2006, 103:11613-11618.

37. Eldar A, Dorfman R, Weiss D, Ashe H, Shilo BZ, Barkai N. Robustness of the BMP morphogen gradient in Drosophila embryonic patterning. Nature 2002, 419:304-308.

38. Mizutani CM, Nie Q, Wan FY, Zhang YT, Vilmos P, Sousa-Neves R, Bier E, Marsh JL, Lander AD. Formation of the BMP activity gradient in the Drosophila embryo. Dev Cell 2005, 8:915-924.

39. Eldar A, Shilo BZ, Barkai N. Elucidating mechanisms underlying robustness of morphogen gradients. Curr Opin Genet Dev 2004, 14:435-439.

40. Boettiger AN, Levine M. Synchronous and stochastic patterns of gene activation in the Drosophila embryo. Science 2009, 325:471-473.

41. Crick FH. Diffusion in embryogenesis. Nature 1970, 225:420-422.

42. Lander AD, Nie W, Wan FY. Do morphogen gradients arise by diffusion? Dev Cell 2002, 2:785-796.

43. Berezhkovskii AM, Sample C, Shvartsman SY. How long does it take to establish a morphogen gradient? Biophys J 2010, 99:L59-61.

44. Gordon P, Sample C, Berezhkovskii AM, Muratov CB, Shvartsman SY. Local kinetics of morphogen gradients. Proc Natl Acad Sci 2011, 108:6157-6162.

45. Berezhkovskii AM, Sample C, Shvartsman SY. Formation of morphogen gradients: local accumulation time. Phys Rev E 2011, 83:051906.

46. Little SC, Tkačik G, Kneeland TB, Wieschaus EF, Gregor $\mathrm{T}$. The formation of the Bicoid morphogen gradient requires protein movement from anteriorly localized mRNA. PLoS Biol 2011, 9:e1000596.

47. Grimm O, Coppey M, Wieschaus E. Modelling the Bicoid gradient. Development 2010, 137:2253-2264.

48. Drocco JA, Grimm O, Tank DW, Wieschaus EF. Measurement and perturbation of morphogen lifetime: Effects on gradient shape. Biophys J 2011, 101:1807-1815

49. Li WX. Functions and mechanisms of receptor tyrosine kinase Torso signaling: lessons from Drosophila embryonic terminal development. Dev Dyn 2005, 232:656-672.

50. Stein D, Stevens LM. The torso ligand, unmasked? Sci STKE 2001, 4:PE2 (Review).

51. Lloyd TE, Atkinson R, Wu MN, Zhou Y, Pennetta G, Bellen HJ. Hrs regulates endosome membrane invagination and tyrosine kinase receptor signaling in Drosophila. Cell 2002, 108:261-269.

52. Sprenger F, Nüsslein-Volhard C. Torso receptor activity is regulated by a diffusible ligand produced at the extracellular terminal regions of the Drosophila egg. Cell 1992, 71:987-1001.

53. Casanova J, Struhl G. The torso receptor localizes as well as transduces the spatial signal specifying terminal body pattern in Drosophila. Nature 1993, 362:152-155.

54. Chen Y, Struhl G. Dual roles for patched in sequestering and transducing hedgehog. Cell 1996, 87:553-563.

55. Berezhkovskii AM, Coppey M, Shvartsman SY. Signaling gradients in cascades of two-state reaction-diffusion systems. Proc Natl Acad Sci 2009, 106:1087-1092.

56. Coppey M, Boettiger AN, Berezhkovskii AM, Shvartsman SY. Nuclear trapping shapes the terminal gradient in the Drosophila embryo. Curr Biol 2008, 18:915-919.

57. Kim Y, Coppey M, Grossman R, Ajuria L, Jimenez G, Paroush Z, Shvartsman SY. MAPK substrate competition integrates patterning signals in the Drosophila embryo. Curr Biol 2010, 20:446-451.

58. Watson JD, Baker TA, Bell SP, Gann A, Levine M, Losick R. Molecular Biology of the Gene. Cold Spring Harbor: Benjamin Cummings; 2007.

59. Liang HL, Nien CY, Liu HY, Metzstein MM, Kirov N, Rushlow C. The zinc-finger protein Zelda is a key activator of the early zygotic genome in Drosophila. Nature 2008, 456:400-U467.

60. Liberman LM, Stathopoulos A. Design flexibility in cisregulatory control of gene expression: synthetic and comparative evidence. Dev Biol 2009, 327:578-589.

61. Bintu L, Buchler NE, Garcia HG, Gerland U, Hwa T, Kondev J, Kuhlman T, Phillips R. Transcriptional regulation by the numbers: applications. Curr Opin Genet Dev 2005, 15:125-135.

62. Dresch JM, Liu XZ, Arnosti DN, Ay A. Thermodynamic modeling of transcription: sensitivity analysis 
differentiates biological mechanism from mathematical model-induced effects. BMC Syst Biol 2010, 4:35-45.

63. Fakhouri WD, Ay A, Sayal R, Dresch J, Dayringer E, Arnosti DN. Deciphering a transcriptional regulatory code: modeling short-range repression in the Drosophila embryo. Mol Syst Biol 2009,6:article 341.

64. Ochoa-Espinosa A, Yu D, Tsirigos A, Struffi P, Small S. Anterior-posterior positional information in the absence of a strong Bicoid gradient. Proc Natl Acad Sci 2009, 106:3823-3828.

65. Löhr U, Chung H-R, Beller M, Jäckle H. Antagonistic action of Bicoid and the repressor Capicua determines the spatial limits of Drosophila head gene expression domains. Proc Natl Acad Sci 2009, 106:21695-21700.

66. Alon U. Network motifs: theory and experimental approaches. Nat Rev Genet 2007, 8:450-461.

67. Lewis J, Slack JM, Wolpert L. Thresholds in development. J Theor Biol 1977, 65:579-590.

68. Gardner TS, Cantor CR, Collins JJ. Construction of a genetic toggle switch in Escherichia coli. Nature 2000, 403:339-342.

69. Acloque H, Ocaña OH, Matheu A, Rizzoti K, Wise C, Lovell-Badge R, Nieto MA. Reciprocal repression between Sox3 and snail transcription factors defines embryonic territories at gastrulation. Dev Cell 2011, 21:546-558.

70. Saka Y, Smith JC. A mechanism for the sharp transition of morphogen gradient interpretation in Xenopus. BMC Dev Biol 2007, 7:47.

71. Kim Y, Andreu JM, Lim B, Chung K, Terayama M, Berg CA, Jimenez G, Lu H, Shvartsman SY. Gene regulation by MAPK substrate competition. Dev Cell 2011, 20:880-887.

72. Papatsenko D, Levine M. The Drosophila gap gene network is composed of two parallel toggle switches. PloS One 2011, 6:e21145.

73. Papatsenko D, Goltsev Y, Levine M. Organization of developmental enhancers in the Drosophila embryo. Nucleic Acids Res 2009, 37:5665-5677.

74. Zeitlinger J, Zinzen RP, Stark A, Kellis M, Zhang HL, Young RA, Levine M. Whole-genome ChIP-chip analysis of dorsal, twist, and snail suggests integration of diverse patterning processes in the Drosophila embryo. Gene Dev 2007, 21:385-390.

75. Clyde DE, Corado MS, Wu X, Paré A, Papatsenko D, Small S. A self-organizing system of repressor gradients establishes segmental complexity in Drosophila. Nature 2003, 426:849-853. 